\title{
Plasmon induced NIR response of thin-film a-Si:H solar cells
}

\author{
Florian Lükermann ${ }^{a}$, Ulrich Heinzmann ${ }^{a}$ and Helmut Stiebig ${ }^{a, b}$ \\ ${ }^{a}$ Molecular and Surface Physics, University of Bielefeld, Universitätsstr. 25, D-33615 Bielefeld, \\ Germany; \\ ${ }^{b}$ Malibu GmbH \& Co. KG, Böttcherstr. 7, D-33609 Bielefeld, Germany
}

\begin{abstract}
The defect absorption in hydrogenated amorphous silicon (a-Si:H) photosensitive devices in the presence of resonant absorbing silver nanoparticles (Ag NPs) is investigated. Defect states are created in the a-Si:H network by the incorporated Ag NPs in their direct environment. The strong electromagnetic fields accompanied by the localized surface plasmon (LSP) resonance of the Ag NPs enable high transition rates between the defect states and the conduction band. This results in an observable signal for near infrared (NIR) photon energies in external quantum efficiency (EQE) measurements. By applying different Ag NP size distributions to the devices the LSP resonance is shifted together with a shift of the EQE peak observed for NIR energies. This indicates that the available defect states in the a-Si:H bandgap are addressed by the LSP resonance. Dominant transitions take place for electrons occupying defect levels having an energetic position equal to the LSP resonance energy. Boron doping of the a-Si:H environment shifts the Fermi level towards or below the introduced defect states. These are depleted and cannot contribute to sub bandgap photon absorption. This is an indication for the consistence of the proposed model for resonant defect absorption of the bandgap defect states.
\end{abstract}

Keywords: localized surface plasmon resonance, silver nanoparticles, hydrogenated amorphous silicon, sub bandgap photocurrent, impurity photovoltaic effect, resonant defect absorption

\section{INTRODUCTION}

Efficient thin film silicon solar cell devices rely on the utilization of a broad portion of the solar spectral radiation. Especially wasting of sub bandgap photons is a major loss mechanism for solar cells. The quasi direct bandgap of hydrogenated amorphous silicon (a-Si:H) at $1.7 \mathrm{eV}$ enables strong absorption for the visible spectral region. While red and near infrared (NIR) light is not absorbed effectively. ${ }^{1}$

To take advantage of a broader spectral range different approaches are made. Tandem or multi-junction solar cells composed of a-Si:H, amorphous silicon germanium (a-SiGe:H) based alloys, micro-crystalline silicon ( $\mu c-$ $\mathrm{Si}: \mathrm{H})$ and combinations thereof are investigated. ${ }^{2-4}$ These materials in conjunction cover a broader range of the solar spectrum due to their diverse bandgaps. A different approach is the use of sub bandgap light by photon up-conversion ${ }^{5,6}$ where low energy photons are transformed to high energy photons that can excite electronic transitions between the band edges of the absorber. Here the up converter is electrically isolated from the absorber layer. Similar to this approach is the impurity photovolatic effect (IPV) proposed by Wolf. ${ }^{7}$ This effect enables the excitation from the valence to the conduction band by a two step excitation via a defect state in the bandgap of the absorber. ${ }^{8}$ Theoretical studies predict a benefit for the performance of solar cells by making use of the IPV effect, ${ }^{9,10}$ also in amorphous silicon solar cells. ${ }^{11}$ However, the benefit of this effect is discussed controversial by others, ${ }^{12,13}$ mainly due to increased recombination via the defect levels.

Beside this, the application of metal nanostructures to solar cell devices has found increasing interest for enhancing the photon absorption. Here localized surface plasmon (LSP) resonances that are excited upon interaction between electromagnetic radiation with the metal nanostructures are investigated. Mostly the ability of large metal nanostructures (in the range of hundreds of nanometers) to scatter light is used in order to enhance the optical thickness of solar absorber layers. ${ }^{14-17}$ Apart from this light trapping approach dominated by scattering, the use of small silver nanoparticles (Ag NPs) is investigated. It has been demonstrated in previous contributions

Further author information: Send correspondence to Florian Lükermann E-mail: florian.luekermann@physik.uni-bielefeld.de, Telephone: +49 (0)521 1065471

Next Generation (Nano) Photonic and Cell Technologies for Solar Energy Conversion III, edited by Loucas Tsakalakos, Proc. of SPIE Vol. 8471, 84710S - C 2012 SPIE CCC code: $0277-786 / 12 / \$ 18 \cdot$ doi: $10.1117 / 12.928684$ 
that the application of approximately $20 \mathrm{~nm} \mathrm{Ag} \mathrm{NPs} \mathrm{to} \mathrm{the} \mathrm{interface} \mathrm{of} \mathrm{Schottky} \mathrm{structures} \mathrm{composed} \mathrm{of} \mathrm{a-Si:H}$ and transparent conductive oxide (TCO) layers ${ }^{18-20}$ or inside the intrinsic layer of a-Si:H n-i-p structures ${ }^{20}$ yields a photocurrent for photon energies below the bandgap of the a-Si:H absorber. This is assigned to the creation of defect states in the a-Si:H material due to the presence of the silver nanoparticles in the a-Si:H network, implicitly interface defects or impurities caused by silver atoms. These states lie within the a-Si:H bandgap. Because the defects are created in direct vicinity of the strongly resonant absorbing Ag NPs, they are exposed to the strong electromagnetic fields in their environment ${ }^{21}$ going along with the LSP resonances. These strong fields promote a resonant defect absorption with high transition rates between the created defect states and the band edges. Therefore this finding is a demonstration for the use of the IPV effect in a-Si:H devices. ${ }^{20}$

In this contribution the influence of the Ag NP size distribution and the Ag NP environment on the generated photocurrent for photon energies in the NIR is investigated, especially with respect to doping of a-Si:H in the NP environment. The varying boron doping concentration is expected to shift the Fermi level towards the valence band and should probe the energetic position as well as the nature of the IPV introduced defect states.

\section{EXPERIMENTS}

Ag NPs are incorporated in a-Si:H photosensitive devices (see Fig. 1 (d) and Fig. 2 (a)). These are composed of a front TCO, thin a-Si:H absorber layers (n-i layer system) and a back contact. The thin intrinsic absorber layer $(30 \mathrm{~nm})$ enables a strong build in electrostatic field for efficient charge carrier extraction.

Thin film deposition is mainly carried out in industry sized deposition facilities on glass substrates with a dimension of $1.4 \mathrm{~m}^{2}$. Fabricated devices are composed of a glass substrate with a front TCO, either a $1 \mu \mathrm{m}$ thick sputter deposited Al:ZnO (AZO) or a standard Asahi $\mathrm{SnO}_{2}$. The a-Si:H layers are deposited by plasma enhanced chemical vapor deposition (PECVD). The Ag NPs are prepared by sputtering of silver films with a few nanometers thickness. Afterwards the silver films are annealed in vacuum at temperatures between $150{ }^{\circ} \mathrm{C}$ and $200{ }^{\circ} \mathrm{C}$ for $30 \mathrm{~min}$, resulting in the agglomeration of $\mathrm{Ag}$ NPs caused by surface tension. The silver films itself are deposited by DC magnetron sputtering on the large $1.4 \mathrm{~m}^{2}$ substrate at room temperature and a pressure of $10^{-3}$ mbar, or by dividing the substrate in $5 \times 5 \mathrm{~cm}^{2}$ samples prior to deposition and afterwards sputtering the silver films in an ultra high vacuum chamber at similar deposition conditions. In all cases, the NP size distribution can be altered by varying the silver film thickness. The NP films are characterized using a Zeiss DSM 982 Gemini scanning electron microscope (SEM) with an accelerating voltage of $10 \mathrm{kV}$. From these measurements Ag NP size distributions can be evaluated and can be used for the calculation of extinction spectra for these particles, giving information about the LSP resonances. The calculation of extinction spectra is performed with the open access program MiePlot, ${ }^{22}$ which uses the algorithm for Mie scattering of Bohren and Huffman. ${ }^{23}$ This algorithm is valid for spherical particles. Although the NPs in our investigation are more oblate shaped the overall trend can be used for comparing experimental observations with calculations. The program calculates extinction spectra for each discrete particle size of the used size dispersion histogram and combines them to an overall response.

The back contact applied in all devices is a combination of sputter deposited AZO and silver reflector layers with thicknesses of $80 \mathrm{~nm}$ and $100 \mathrm{~nm}$, respectively. Devices with an active cell area of $1 \mathrm{~cm}^{2}$ are defined by laser scribing.

Different photosensitive a-Si:H device structures are prepared. Schottky type samples in the TCO/AgNP/in/TCO/Ag-reflector configuration (FIG. 1 (d)) are prepared. Furthermore the impact of the Fermi level position on the defect states is investigated by using devices based on the same design. In contrast the doping concentration in the Ag NP environment is varied for different samples from undoped to highly p-doped. The devices are consequently composed of a p-i-n layer system (Fig. 2 (a)). The p-type doping is realized by adding Trimethylborane $\mathrm{B}\left(\mathrm{CH}_{3}\right)_{3}(\mathrm{TMB})$ to the PECVD gas mixture. The Fermi level $\left(\mathrm{E}_{\mathrm{F}}\right)$ position with respect to the different p-doping concentrations is deduced by measuring the dark conductivity of single layers. For this purpose a-Si:H layers with the same doping gas fluxes used for the cell experiments are deposited on quartz glass substrates with a layer thickness of approximately $250 \mathrm{~nm}$ (Fig. 2 (c)). Afterwards coplanar metal electrodes are deposited on top of these layers by thermal evaporation. The samples are then placed in a high vacuum cryostat and heated to $150{ }^{\circ} \mathrm{C}$ prior to conductivity measurement for at least one hour in order to remove surface adsorbates and the influence of the Staebler-Wronski effect. ${ }^{24}$ The dark conductivity $\sigma_{\mathrm{d}}$ is measured in the coplanar contact configuration at several temperatures from $150{ }^{\circ} \mathrm{C}$ downwards. For intrinsic or n-type a-Si:H $\sigma_{\mathrm{d}}$ can be written in the 
form $\sigma_{\mathrm{d}}(\mathrm{T})=\sigma_{0} \exp \left(-\frac{\left(\mathrm{E}_{\mathrm{C}}-\mathrm{E}_{\mathrm{F}}\right)}{\mathrm{kT}}\right)$ or the logarithm $\mathrm{k} \cdot \ln \sigma_{\mathrm{d}}(\mathrm{T})=\mathrm{k} \cdot \ln \sigma_{0}-\frac{\left(\mathrm{E}_{\mathrm{C}}-\mathrm{E}_{\mathrm{F}}\right)}{\mathrm{T}}$. Here $\mathrm{E}_{\mathrm{C}}$ is the conduction band energy, $\mathrm{k}$ is the Boltzmann constant, $\mathrm{T}$ is the temperature in Kelvin and $\sigma_{0}$ is a conductivity prefactor. By plotting $\mathrm{k} \cdot \ln \sigma_{\mathrm{d}}$ versus $1 / \mathrm{T}$ and fitting the data by a straight line, one obtains the difference between conduction band and Fermi level $\left(E_{C}-E_{F}\right)$ as negative slope of the fit. For p-doped samples this procedure results in the distance of $E_{F}$ to the valence band $\left(E_{F}-E_{V}\right){ }^{25}$ To calculate in each case the opposite value, i.e. $\left(E_{F}-E_{V}\right)$ for instrinsic or $\left(E_{\mathrm{C}}-\mathrm{E}_{\mathrm{F}}\right)$ for p-doped samples one has to subtract the obtained value from the bandgap energy. The optical bandgap is calculated from UV-Vis measurements via Tauc's method ${ }^{26}$ by plotting $\sqrt{\operatorname{Eh} \nu}$ versus $\mathrm{h} \nu$ and extrapolating the linear part towards the abscissa.

\section{RESULTS AND DISCUSSION}

In this Section the nature of the defect states inside the a-Si:H bandgap is investigated in more detail. The LSP resonance provides strong fields in the NP environment at the defect locations leading to high transition rates from the defect states. To investigate the influence of an energetic variation of the LSP resonance position on the generated photocurrent the Ag NP size dispersion is altered. Additionally the influence of the Fermi level with respect to the defect states is investigated by introducing a p-doped layer in direct neighborhood to the NPs.

\subsection{Influence of Ag NP size on resonant defect absorption}

Fig. 1 (a)-(c) shows silver films of varying layer thickness after annealing, deposited onto a plain AZO layer. Increasing the silver layer thickness leads to an increase of the average particle diameter, with a broad size distribution. For the shown examples particle agglomerates of $(10 \pm 5) \mathrm{nm},(23 \pm 6) \mathrm{nm}$ and $(30 \pm 6) \mathrm{nm}$ particle diameter were fabricated by varying the initial Ag layer thickness between 3,5 and $7 \mathrm{~nm}$, respectively. The mean particle size and standard deviation is determined from gaussian functions fitted to the NP distribution histograms. For the incorporation of Ag NPs in a-Si:H photosensitive device structures (Fig. 1 (d)), where the dielectric environment is mainly dominated by the over-coated silicon absorber with a refractive index of around $4,{ }^{1}$ LSP resonances in the near infrared between 800 and $900 \mathrm{~nm}$ can be expected. The external quantum efficiency (EQE) measured at $0 \mathrm{~V}$ is illustrated in Fig. 1 (e). Additionally the EQE of a device without NPs is plotted. A maximum EQE amplitude of around 50\% is measured due to the very thin i-layer thickness. In the visible range below $700 \mathrm{~nm}$, where the light absorption of a-Si:H is efficient, the device with NPs shows a decreased photocurrent. This is attributed to damped oscillations of the conduction band electrons inside the $\mathrm{Ag}$ NPs, that dissipates energy to heat and reduces the amount of transmitted energy to the a-Si:H absorber. For wavelength larger than $750 \mathrm{~nm}$, corresponding to the a-Si:H bandgap, no photocurrent can be measured for the device without NPs. In contrast to a significant photocurrent signal observed for the devices with NPs. The measurements, shown in the inset in Fig. 1 (e) with an enlarged ordinate scale, exhibit well defined peaks in the NIR. These peaks are red shifted with an increasing particle size, while the amplitude of the peaks decreases. Mie calculations on the LSP resonances for the applied silver NP dispersions of Fig. 1 (a)-(c) in a silicon environment $(\mathrm{n}=4)$ are performed, owing to the dominant influence of the over-coated a-Si:H layers. The calculated, normalized extinction spectra are illustrated in Fig. 1 (e) top. Increasing the mean particle size of the NP dispersion a red shift of the LSP resonance is observed. The calculated LSP spectra show a good agreement with the measured EQE curves for samples with the according particle distributions, as indicated by the dashed vertical lines in the insets. Hence a shift of the LSP resonance is accompanied by a shift of the photocurrent response. This can be understood in terms of the proposed defect absorption model. ${ }^{20}$ Supposing the defect states created by the presence of the Ag NPs in the a-Si:H network are broadly distributed in the a-Si:H bandgap, as indicated by the broad EQE signal up to $1100 \mathrm{~nm}$. And taking into account that the strong fields associated to the resonances are necessary for high transition rates, the most effective excitation takes place for photon energies equal to the LSP resonance position. Because in these a-Si:H i-n structures electron transport is dominating, the LSP resonance energy determines the energetic distance of the conduction band with respect to the defect states that contribute the most to the transitions. By changing the LSP resonance energy defect levels with a different energetic distance from the conduction band are addressed. The defect states are effectively scanned by varying the LSP resonance energy.

Proc. of SPIE Vol. 847184710 S-3 

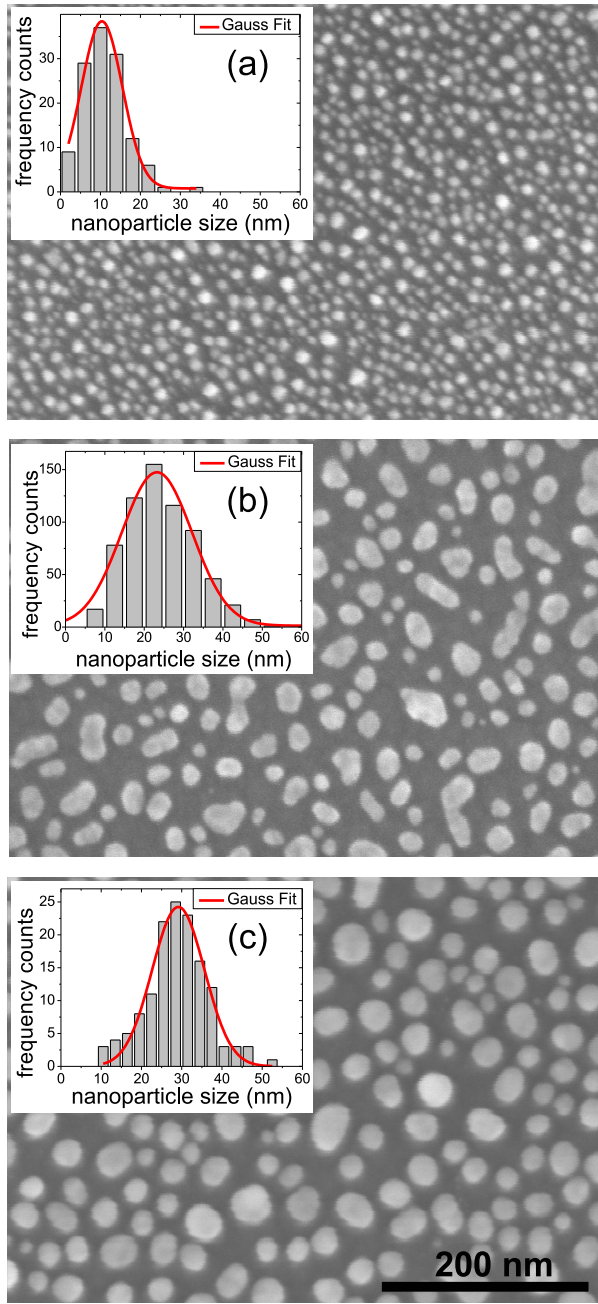

(d)
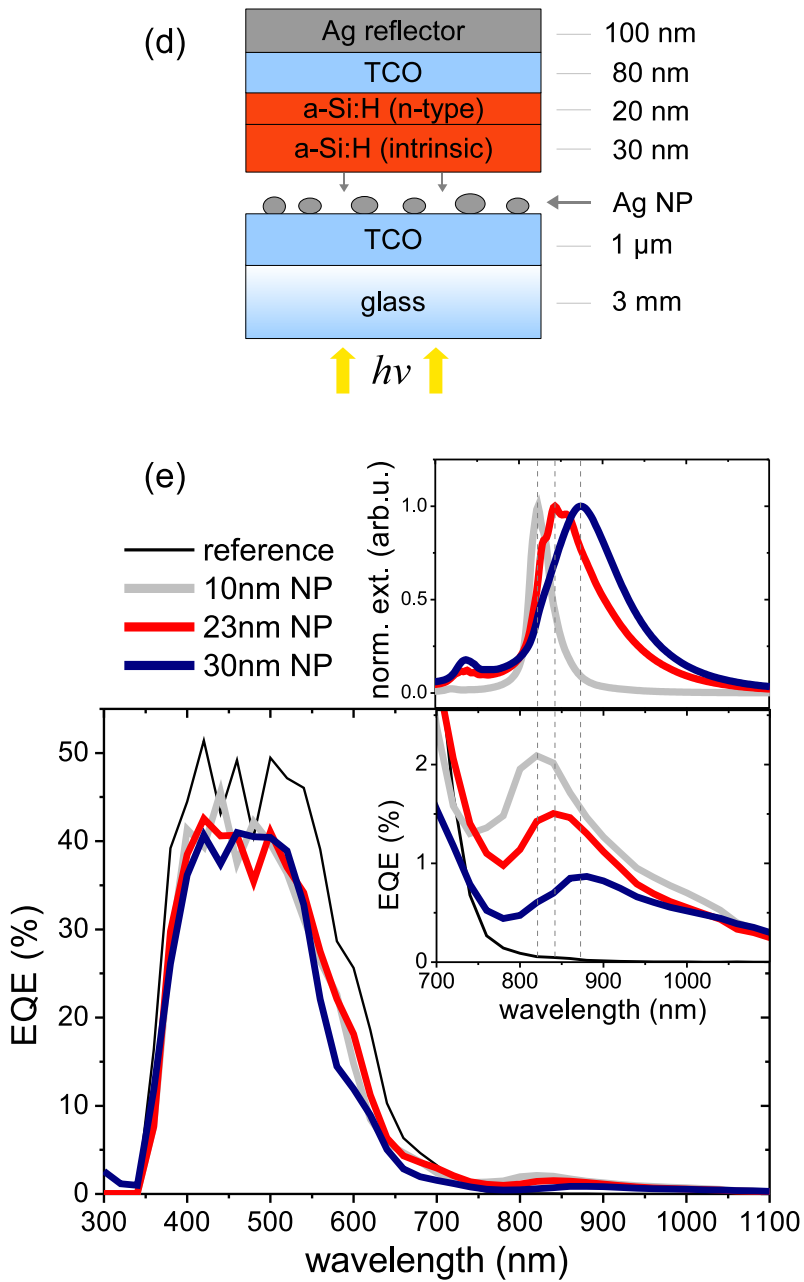

Figure 1. (a), (b), (c) Scanning electron microscope images of $3 \mathrm{~nm}, 5 \mathrm{~nm}$ and $7 \mathrm{~nm}$ silver films after annealing (same scale for all images), deposited on smoth AZO surface, corresponding to average particle diameters of (10 \pm 5$) \mathrm{nm},(23 \pm 6) \mathrm{nm}$ and $(30 \pm 6) \mathrm{nm}$, respectively. The according particle size distributions are shown alongside. (d) Schematic design of photosensitive device with incorporated Ag NPs, in the TCO/Ag NP/a-Si:H i-n/TCO configuration (not true to scale). (e) EQE measurements of these photosensitive devices with incorporated Ag NP dispersions shown in (a)-(c). The EQE signal in the NIR range is shown in the inset with an enlarged ordinate scale. The inset on top shows the calculated normalized extinction of Ag NPs with the size distributions of (a)-(c) in a silicon environment with a refractive index of $\mathrm{n}=4$. Dashed vertical lines indicate the overlap of the calculated LSP resonances with the observed EQE peaks.

The decrease of the EQE peak amplitude in the NIR with increasing particle size might be attributed to the overall transition mechanism. The transition of an electron from the valence to the conduction band via a defect state requires effectively two excitations. Beneath the excitation by an NIR photon from a defect level to the conduction band, an additional excitation step presumably by thermal activation from the valence band to the defect level is required. The larger the NPs are, the smaller the LSP resonance energy and the smaller the energetic distance of defect states with dominant transitions to the conduction band. Thus defect levels with a larger distance to the valence band contribute to the resonant transitions. This leads to a decreasing probability for thermal activation of electrons from the valence band to these defect levels. The consequence is a decreasing efficiency for the overall transition. 
(a)
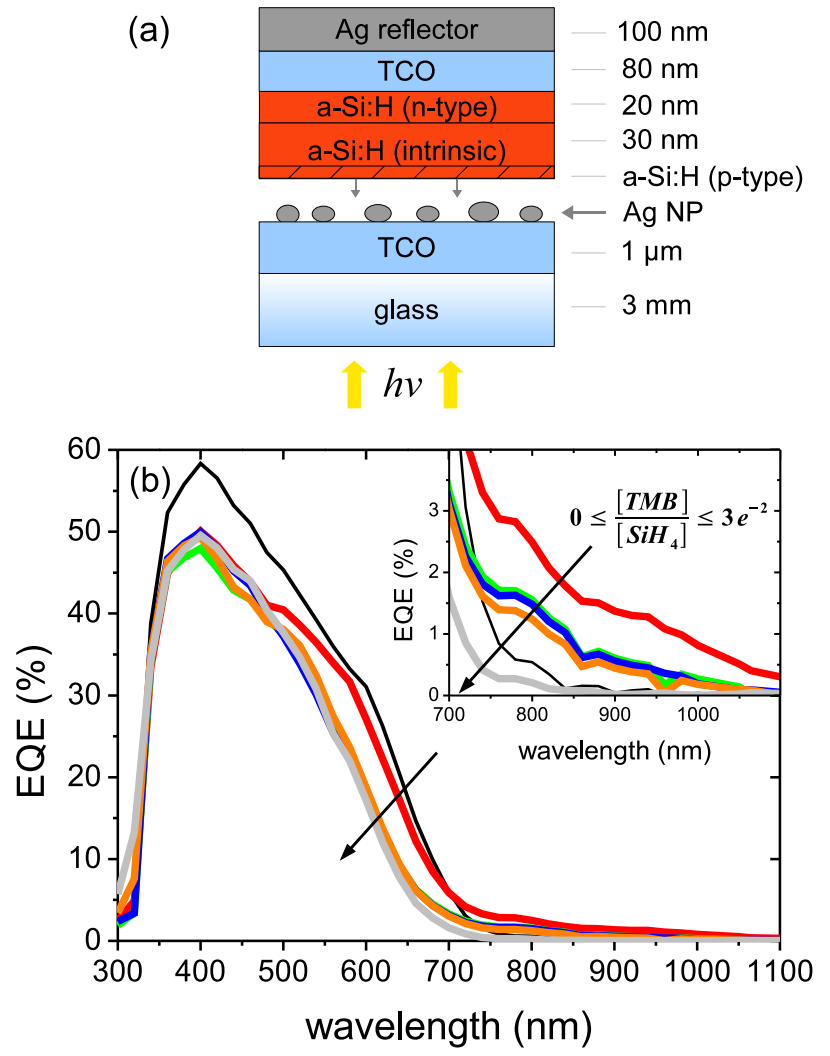

(c)
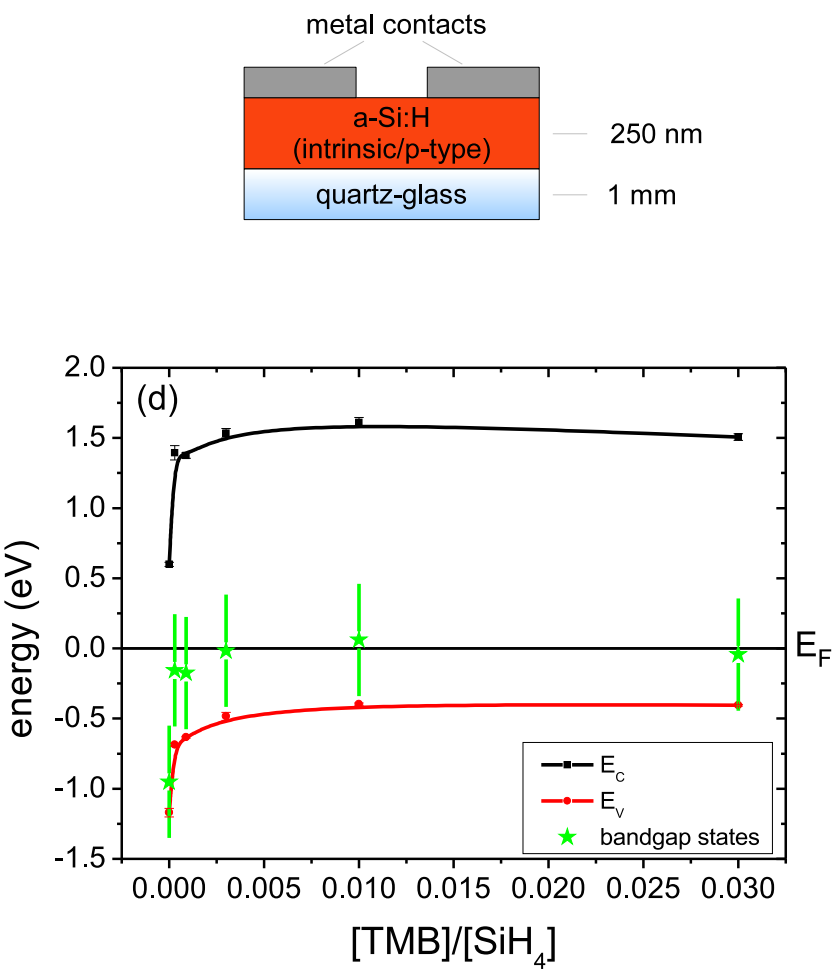

Figure 2. (a) Schematic design of TCO/Ag NP/a-Si:H p-i-n/TCO Schottky structures with a varying doping concentration from intrinsic to highly p-doped at the front TCO/a-Si:H interface (not true to scale). Thus thin p-i-n structures are

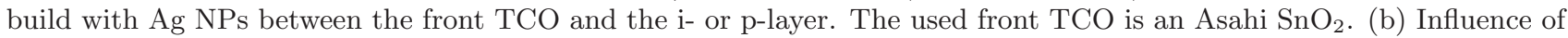
doped environment of Ag NPs on EQE, the inset illustrates the characteristics in the NIR range with an enlarged ordinate scale. The thin black line shows the i-n reference without Ag NPs, while the thick colored lines represent samples with $\mathrm{Ag}$ NPs. The tendency of an increased doping concentration in the gas mixture varying between $[\mathrm{TMB}] /[\mathrm{SiH} 4]=0(\mathrm{i}-\mathrm{n}$ device) to $[\mathrm{TMB}] /\left[\mathrm{SiH}_{4}\right]=3 \mathrm{e}^{-2}$ (highly p-doped p-i-n device) is indicated by the arrows. (c) Schematic sample geometry for measurement of dark conductivity of single a-Si:H layers with varied p-type doping (not true to scale). (d) Position of valence band (red dots) and conduction band (black squares) with respect to the Fermi level (set to $E_{F}=0$ ). The measurement is carried out for single a-Si:H layers with the according doping concentrations used for the cell experiments shown in (b). For visual clarity, solid lines are drawn along the measured data to indicate their trend with p-type doping. The LSP induced bandgap states (green stars) with respect to the conduction band are also shown. The state energy is deduced from the energetic position of the most dominant transition $(\approx 800 \mathrm{~nm} \rightarrow 1.55 \mathrm{eV})$ observed for the EQE measurement of the undoped sample. The errorbars indicate the width of the observable NIR EQE signal especially towards the low energy side $(\approx 0.4 \mathrm{eV})$.

\subsection{Influence of doped environment on resonant defect absorption}

The model for the origin of the sub bandgap excitation by resonant defect absorption from the vicinity of the Ag NPs relies, for the case of an a-Si:H i-n structure, on the fact that the created defect states are occupied by electrons. To investigate the nature of the defect states inside the a-Si:H bandgap and to test the proposed model, the doping in direct environment of the Ag NPs is varied. By this the influence of the Fermi level shift on the defect absorption is investigated. The NPs are deposited in devices similar to that of Section 3.1. In contrast a p-doped (Boron) layer is introduced adjacent to the NPs. Thus a TCO/AgNP/a-Si:H p-i-n/TCO structure is build (Fig. 2 (a)). The doping concentration is varied for different samples by changing the gas flow of Trimethylborane $\mathrm{B}\left(\mathrm{CH}_{3}\right)_{3}$ (TMB) during the p-layer deposition. The ratio of TMB to Silane $\left(\mathrm{SiH}_{4}\right)$ denoted as $[\mathrm{TMB}] /\left[\mathrm{SiH}_{4}\right]$ is varied from 0 (undoped) for the reference sample to $3 \cdot 10^{-2}$ (highly doped). The intrinsic 
layer thickness of the device without p-doped layer was about $30 \mathrm{~nm}$. The introduced p-doped layer in all cases has a thickness of around $5 \mathrm{~nm}$ while the i-layer thickness is reduced to $25 \mathrm{~nm}$ in comparision to the structure shown in Fig. 1. For all a-Si:H based devices, the total absorber layer thickness was kept constant. The front

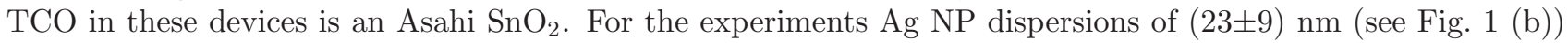
are used.

EQE measurements of the devices are shown in Fig. 2 (b). Owing to the small absorber layer thickness, a maximum EQE signal of 55 to $60 \%$ is measured for the i-n reference device (thin black line) without Ag NPs and without a p-doped layer. For NIR photons above $750 \mathrm{~nm}$ no photocurrent response is measured. By incorporating Ag NPs (thick red curve) to the $\mathrm{i}-\mathrm{n}$ device a decreased signal is observed in the visible range. This is again related to damping losses at the metal nanoparticles.

For photon energies in the NIR (inset in Fig. 2 (b)) two main findings can be made. First, the incorporation of Ag NPs leads to a significant EQE signal. In this case no distinct peak is observed, however a dominant shoulder at around $800 \mathrm{~nm}$ becomes evident. Thus the main contribution of defect absorption is shifted towards higher energies closer to the bandbap energy. The second finding is a decreasing EQE signal with increasing p-layer doping in the NP environment. The trend of EQE with an increasing doping concentration for samples with $\mathrm{Ag}$ NPs (thick curves) is indicated by the black arrows. The sample with highly doped p-layer shows a lower signal than the i-n reference device without Ag NPs. This trend is also evident for visible wavelengths above $500 \mathrm{~nm}$ where a decreased EQE signal is observed for the p-doped samples with respect to the undoped i-n device.

The shift of the main NIR EQE signal to higher energies might be related to the fact that in contrast to the samples discussed in Section 3.1 deposited on smooth AZO layers (Fig. 1 (e)), these samples are deposited on rough Asahi $\mathrm{SnO}_{2}$. This increases the influence of the rough underlying $\mathrm{SnO}_{2}$ layer on the dielectric environment of the NPs. In consequence a shift of the plasmon resonance might shift the main EQE contribution to higher energies. In addition scattering at the rough interface could have an influence.

The decreasing EQE signal with increasing p-type doping in the NP environment could be related to a shift of the Fermi level below the bandgap states. Therfore the Fermi level position in p-doped a-Si:H layers is measured as described in Section 2 with the used doping gas ratios. Fig. 2 (d) shows the distance of the valence and conduction band with respect to the Fermi level, which is set to zero in this diagram. The Tauc bandgap as the difference between $\mathrm{E}_{\mathrm{C}}$ and $\mathrm{E}_{\mathrm{V}}$ has a value of $1.75 \mathrm{eV}$ for the intrinsic sample. For the lowest p-doping concentration the largest bandgap of around $2.1 \mathrm{eV}$ is measured, it decreases with increasing doping concentration to $1.9 \mathrm{eV}$ mainly due to the role of $\mathrm{H}_{2}$ incorporation. The position of accessible bandgap states (green stars) is also shown. Their energetic position is deduced from the main contribution observed in the EQE measurement for the case of the i-n device with incorporated $\mathrm{Ag}$ NPs at roughly $800 \mathrm{~nm}(1.55 \mathrm{eV})$. It is plotted at each doping concentration relative to the conduction band. The error-bars indicate the width of the observable NIR EQE signal of around $0.4 \mathrm{eV}$ especially towards the low energy region (up to $1100 \mathrm{~nm}(1.1 \mathrm{eV})$ ). With an increased doping concentration a shift of the Fermi level towards the valence band and the defect states is observed. For medium and high doping concentrations the low energy part of the gap states is already shifted above $\mathrm{E}_{\mathrm{F}}$ leading to a depletion of these states. This agrees with the observation of a decreasing EQE signal in the NIR, but cannot explain the complete disappearance of the NIR signal for the highest doping concentration $\left([\mathrm{TMB}] /[\mathrm{SiH} 4]=3 \mathrm{e}^{-2}\right)$. This would require the Fermi level to shift completely below the accessible states. The measured Fermi level position for high doping concentrations might has to be reduced due to the statistical shift by roughly $200 \mathrm{meV}{ }^{25}$ The Fermi level would accordingly shift below the bandgap states leading to a depletion and consequently to a lack of an observable EQE signal for photons with sub bandgap energy. In any case the observed EQE NIR signal is sensitive to a shift of the Fermi level towards the bandgap states. This is in agreement with the proposed defect states in the a-Si:H network due to the presence of the Ag NPs. When these states are depleted NIR photons cannot elevate electrons to the conduction band.

In addition other reasons could be of importance. The introduced doping increases the defect density by orders of magnitude ${ }^{27}$ and leads to increased recombination of charge carriers. Especially for those generated inside the p-layer, i.e. for electrons originating from the sub bandgap transitions near the NPs. Furthermore the NPs are outside the build in electrostatic field of the p-i-n diode. With an increased doping concentration the electrons generated in direct vicinity of the Ag NPs can be transported less efficient towards the n-layer. 


\section{CONCLUSIONS}

The effect of resonant defect absorption in a-Si:H photosensitive devices in the presence of Ag NPs is investigated. In particular the role of a varying Ag NP size dispersion is studied, as well as the influence of the NP environment with respect to doping.

Altering of the Ag NP size dispersion leads to a shift of the LSP resonance condition. This shift is reflected in the observed EQE signal for photon energies below the bandgap of the a-Si:H material. This indicates a correlation of the defect absorption and the plasmon resonance by providing strong electromagnetic fields at the defect sites in the vicinity of the NPs and enables high transition rates. By changing the LSP resonance energy, different defect levels are addressed. Thus the defect states are energetically scanned by the LSP resonance, according to transitions between defect levels and the conduction band having a distance equal to the LSP resonance energy. Introducing a p-type doping in direct environment of the Ag NPs leads to a decrease of the NIR EQE signal. One explanation for this observation could be the following. The introduced p-doping leads to a Fermi level shift towards the valence band resulting in a depletion of available defect states. Accordingly transitions from these states are not possible. This finding is an indication for the consistence of the proposed model of the resonant absorption from defect states close to the NPs.

The use of the sub bandgap defect absorption for solar cell applications seems to be limited in terms of the overall losses in the visible spectral region that are not overcompensated by the photocurrent for NIR photon energies. However the strong difference of the signal in the NIR between devices with and without Ag NPs in combination with the possibility to control the strength and spectral position by changing the NP size might be of interest for example in NIR detector applications. Additionally the freedom to take advantage of this effect

in Schottky type devices or alternatively in p-i-n diodes ${ }^{20}$ where the particles are embedded inside the intrinsic layer could be of interest in this context.

\section{REFERENCES}

1. A. Shah, H. Schade, M. Vanecek, J. Meier, E. Vallat-Sauvain, N. Wyrsch, U. Kroll, C. Droz, and J. Bailat, "Thin-film silicon solar cell technology," Progress in photovoltaics: Research and applications 12(2-3), pp. 113-142, 2004.

2. H. Keppner, J. Meier, P. Torres, D. Fischer, and A. Shah, "Microcrystalline silicon and micromorph tandem solar cells," Applied Physics A: Materials Science \& Processing 69, pp. 169-177, 1999. $10.1007 / \mathrm{s} 003390050987$.

3. X. Deng, X. Liao, S. Han, H. Povolny, and P. Agarwal, "Amorphous silicon and silicon germanium materials for high-efficiency triple-junction solar cells," Solar Energy Materials and Solar Cells 62(12), pp. 89 - 95, 2000 .

4. A. Shah, J. Meier, E. Vallat-Sauvain, N. Wyrsch, U. Kroll, C. Droz, and U. Graf, "Material and solar cell research in microcrystalline silicon," Solar Energy Materials and Solar Cells 78(14), pp. 469 - 491, 2003.

5. T. Trupke, M. A. Green, and P. Würfel, "Improving solar cell efficiencies by up-conversion of sub-band-gap light," J. Appl. Phys. 92(7), pp. 4117-4122, 2002.

6. B. Richards and A. Shalav, "Enhancing the near-infrared spectral response of silicon optoelectronic devices via up-conversion," Electron Devices, IEEE Transactions on 54, pp. 2679 -2684, oct. 2007.

7. M. Wolf, "Limitations and possibilities for improvement of photovoltaic solar energy converters: Part i: Considerations for earth's surface operation," Proceedings of the IRE 48, pp. 1246 -1263, july 1960.

8. M. Green, Silicon solar cells: advanced principles 83 practice, Centre for Photovoltaic Devices and Systems, University of New South Wales, 1995.

9. M. Keevers and M. Green, "Efficiency improvements of silicon solar cells by the impurity photovoltaic effect," J. Appl. Phys. 75(8), pp. 4022-4031, 1994.

10. A. Luque and A. Martí, "Increasing the efficiency of ideal solar cells by photon induced transitions at intermediate levels," Phys. Rev. Lett. 78(26), pp. 5014-5017, 1997.

11. H. Matsumura and H. Kasai, "Theoretical study for drastic improvement of solar cell efficiency," Japanese Journal of Applied Physics 34(Part 1, No. 5A), pp. 2252-2259, 1995.

12. W. Shockley and H. J. Queisser, "Detailed balance limit of efficiency of p-n junction solar cells," J. Appl. Phys. 32(3), pp. 510-519, 1961. 
13. S. Z. Karazhanov, "Impurity photovoltaic effect in indium-doped silicon solar cells," J. Appl. Phys. 89(7), pp. 4030-4036, 2001.

14. V. E. Ferry, M. A. Verschuuren, H. B. T. Li, E. Verhagen, R. J. Walters, R. E. I. Schropp, H. A. Atwater, and A. Polman, "Light trapping in ultrathin plasmonic solar cells," Opt. Express 18(102), pp. A237-A245, 2010.

15. T. Temple, G. Mahanama, H. Reehal, and D. Bagnall, "Influence of localized surface plasmon excitation in silver nanoparticles on the performance of silicon solar cells," Solar Energy Materials and Solar Cells 93 , pp. 1978-1985, 2009.

16. S. Pillai, K. R. Catchpole, T. Trupke, and M. A. Green, "Surface plasmon enhanced silicon solar cells," J. Appl. Phys. 101, p. 093105, 2007.

17. F. J. Beck, S. Mokkapati, and K. R. Catchpole, "Plasmonic light-trapping for Si solar cells using selfassembled, Ag nanoparticles," Progress in Photovoltaics: Research and Applications 18, pp. 500-504, 2010.

18. E. Moulin, P. Q. Luo, B. Pieters, J. Sukmanowski, J. Kirchhoff, W. Reetz, T. Müller, R. Carius, F. X. Royer, and H. Stiebig, "Photoresponse enhancement in the near infrared wavelength range of ultrathin amorphous silicon photosensitive devices by integration of silver nanoparticles," Appl. Phys. Lett. 95, p. $033505,2009$.

19. P. Luo, E. Moulin, J. Sukmanowski, F. X. Royer, X. M. Dou, and H. Stiebig, "Enhanced infrared response of ultra thin amorphous silicon photosensitive devices with Ag nanoparticles," Thin Solid Films 517(23), pp. 6256-6259, 2009.

20. F. Lükermann, U. Heinzmann, and H. Stiebig, "Plasmon enhanced resonant defect absorption in thin a-Si:H n-i-p devices," Appl. Phys. Lett. 100(25), p. 253907, 2012.

21. J.-Y. Lee and P. Peumans, "The origin of enhanced optical absorption in solar cells with metal nanoparticles embedded in the active layer," Opt. Express 18, pp. 10078-10087, May 2010.

22. P. Laven, "Mie Plot v4.2 - A computer program for scattering of light from a sphere using Mie theory \& the Debye series." http://philiplaven.com/mieplot.htm, 32010.

23. C. F. Bohren and D. R. Huffman, Absorption and Scattering of Light by Small Particles, Wiley-VCH Verlag GmbH, Weinheim, Germany, 2007.

24. W. Beyer and B. Hoheisel, "Photoconductivity and dark conductivity of hydrogenated amorphous silicon," Solid State Commun. 47(7), pp. 573-576, 1983.

25. A. Shah and C. Droz, Thin-film Silicon: Photovoltaics and Large-area Electronics, EFPL Press, 2009.

26. J. Tauc, R. Grigorovici, and A. Vancu, "Optical properties and electronic structure of amorphous germanium," Physica Status Solidi (b) 15(2), pp. 627-637, 1966.

27. R. A. Street, D. K. Biegelsen, and J. C. Knights, "Defect states in doped and compensated a-Si:H," Phys. Rev. B 24, pp. 969-984, 1981. 